\title{
RDUS
}

Revue de DROIT UNIVERSITÉ DE SHERBROOKE

Titre : $\quad$ LA BONNE FOI À LA FIN DU VINGTIÈME SIÈCLE

Auteur(s): $\quad$ Menezez CORDEIRO

Revue : $\quad$ RDUS, 1995-1996, volume 26, numéro 2

Pages: $\quad$ 223-245

ISSN : $\quad$ 0317-9656

Éditeur: $\quad$ Université de Sherbrooke. Faculté de droit.

URI : $\quad$ http://hdl.handle.net/11143/12866

DOI : https://doi.org/10.17118/11143/12866 
Page vide laissée intentionnellement. 


\section{LA BONNE FOI À LA FIN DU VINGTIÈME SIÈCLE}

par Menezez CORDEIRO*

SOMMAIRE

1. APPLICATIONS PRATIQUES ACTUELLES DE LA BONNE FOI

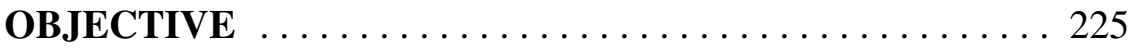

2. LES LECTURES CLASSIQUES . . . . . . . . . . . . . . . 229

3. L'IMPÉRATIF DE LA CONFIANCE ET LA MATÉRIALITÉ SOUS-JACENTE; LES SYSTÈMES DE LA BONNE FOI ET DE

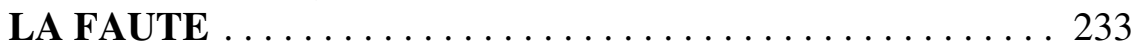

4. TENDANCES RÉCENTES : LA RÉDUCTION DU RÔLE DE LA BONNE FOI . . . . . . . . . . . . . . . . . . . . . 234

5. LA MÉTHODE : UNE AIDE PRATIQUE? ........... 236

6. BILAN : LE RÔLE ACTUEL DE LA BONNE FOI; PROBLÈMES ET PENSÉE SYSTÉMATIQUE . . . . . . . . . . . . . . 239

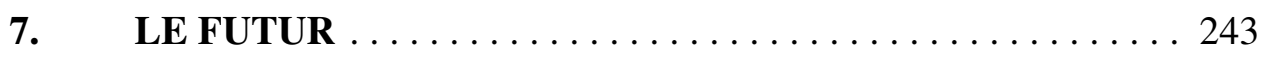

*. Professeur à l'Université de Lisbonne. 


\section{APPLICATIONS PRATIQUES ACTUELLES DE LA BONNE FOI OBJECTIVE}

La bonne foi, en tant que règle de conduite -ou bonne foi objective, dans les langues latines ${ }^{1}$ - connaît un succès généralisé dans les codes civils actuels. Les exemples plus classiques sont, aujourd'hui encore, ceux de l'article 1134 al. 3 du Code civil français de 1804 et de l'article 242 du Code civil allemand de $1896 .^{2}$ Les études qui s'occupent du droit européen des contrats - du reste tout à fait théorique - insistent sur la nécessité de respecter les exigences de la bonne foi. $^{3}$

La bonne foi est un concept indéterminé, empreint d'une grande abstraction. On ne peut en juger sans connaître son application réelle dans les divers droits. Nous commencerons par un examen des décisions des tribunaux. Pour des raisons pratiques, nous allons limiter la recherche aux deux expériences

1. En allemand, l'adjectivation n'est pas nécessaire : la bonne foi objective est dite Treu und Glauben, alors que la bonne foi subjective présente, par exemple, dans le domaine de la prescription est dite guter Glaube.

2. Selon l'article 1134 du Code civil français, «les conventions légalement formées tiennent lieu de loi à ceux qui les ont faites. Elles ne peuvent être révoquées que de leur consentement mutuel, ou pour les causes que la loi autorise. Elles doivent être exécutées de bonne foi». À son tour, l'article 242 du Code civil allemand ou Bürgerliches Gesetzbuch [ci-après : BGB], impose que le débiteur doive exécuter la prestation en considération des usages du trafic. Les codifications tardives contiennent en règle générale des références diverses à la bonne foi objective, en accord avec ses concrétisations. C'est le cas du Code civil italien de 1942 dont l'article 1337 a trait à la bonne foi précontractuelle et l'article 1375 à la bonne foi dans l'exécution des obligations, ou du Code civil portugais de 1966 qui distingue la bonne foi précontractuelle, (article 227) l'abus de droit par opposition à la bonne foi, l'imprévision par exigence de la bonne foi (article 437) et la bonne foi dans l'exécution des obligations (article 762\%). Finalement, les codifications plus récentes : le Code civil hollandais, dans son Livre 6, article 2, a préféré la formule «honnêteté et équité» (redelijkheid en billijkheid); le Code civil du Québec de 1994 pose l'exigence de la bonne foi dans l'exercice des droits (articles 6 et 7), dans la constitution, l'exécution et l'extinction des obligations (article 1375) et le contexte des clauses abusives. Ce dernier est le système le plus parfait à notre connaissance.

3. O. Lando et H. Beale, Principles of European Contract Law, I, (1995), art. 1.104-1.107 (The need to promote good faith and fair dealing); comparé à R. Zimmermann, Konturen eines Europäischen Vertragsrechts, JZ 1995, 477 à la p. 490. On doit remarquer que ces «Principles» ont un style semblable aux «Restatements» des États-Unis d'Amérique. 
les plus marquantes du Continent : l'allemande et la française. ${ }^{4}$ Nous considérerons les décisions publiées lors des deux dernières années.

En Allemagne, la bonne foi trouve son premier champ d'application pratique dans l'imprévision civile, plus particulièrement dans des questions provoquées par la réunification. L'imprévision, appuyée sur la bonne foi, pourrait porter sur des crédits issus d'anciens organismes de l'ex-République démocratique allemande [ci-après ex-RDA $]^{5}$ ou sur des pensions, ${ }^{6}$ mais non sur des rapports de travail, dont l'objet serait devenu impossible. ${ }^{7}$ L'imprévision civile s'est révélée efficace dans des questions concomitantes de l'introduction du Deutsche Mark, dans l'économie est-allemande. ${ }^{8}$ Les contrats antérieurs à la réunification et examinés à la lumière du droit de l'ex-RDA sont jugés valides en ce qui concerne par application de l'imprévision. ${ }^{9}$ Les problèmes de la réunification ont donné encore lieu à d'autres applications : ainsi, la prétention de l'État fédéral allemand de recevoir une provision forcée, pour un représentant obligatoire, dans des opérations d'importation, régies par d'anciennes lois de l'ex-RDA, a été jugée inadmissible contrevenant aux exigences de la bonne foi. ${ }^{10}$ Le fiduciaire à l'origine de la création d'un rapport fiduciaire destiné à détourner les anciennes lois restrictives sur le commerce des devises en ex-RDA, ne peut plus, maintenant, sans contrevenir aux exigences, en invoquer la nullité. ${ }^{11}$ En simplifiant, on peut considérer que, dans le domaine de la réunification, la bonne foi n'a pas trouvé d'application aux dépens, des particuliers. Ainsi, les tribunaux on décidé que les nullités, par défaut de forme, ne pouvaient être bloquées, ${ }^{12}$ l'application de la responsabilité pré-contractuelle ne pouvait faire oublier les

4. L'expérience anglaise ne révèle point de recours particulier au concept de bonne foi : E. Ferraris, «La buona fede negli orientamenti della giurisprudenza inglese», (1995) XCIII RDComm 759.

5. $\quad$ BGH 11 octobre 1994, JZ 1995, 514, note U. Stein, JZ 1995, 516.

6. BGH 25 janvier 1995, BGHZ 129, 1996, 320 à la p. 329, qui mentionne la clausula rebus sic stantibus et l'article 242 du BGB.

7. BAG 24 août 1995, NJW 1996, 476.

8. $\quad$ BGH 6 avril 1995, BGHZ 129, 1996, 236 à la p. 251.

9. BGH 21 septembre 1995, DtZ 1996, 23.

10. BGH 20 septembre 1995, DtZ 1996, 48, JZ 1996, 469, note M. Martinek, JZ 1996, 470.

11. BGH 25 octobre 1995, DtZ 1996, 51.

12. BGH 12 janvier 1996, DtZ 1996, 112. 
normes de l'ex-RDA. ${ }^{13}$ Une situation fiduciaire ne pouvait disparaître au préjudice de tiers. ${ }^{14}$

Encore en Allemagne, nous trouvons un large appel à la bonne foi dans le domaine de conditions générales des contrats. ${ }^{15}$ Les applications plus classiques ont toujours une certaine place et ce, malgré les observations que nous formulerons ci-dessous. On en parle quant à la culpa in contrahendo, ${ }^{16}$ quant à la bonne foi dans le domaine de la réception des déclarations de volonté ${ }^{17}$ dans le cautionnement, ${ }^{18}$ dans les assurances, ${ }^{19}$ dans le bail de financement ${ }^{20}$, dans l'interprétation des titres exécutoires,${ }^{21}$ dans les rapports de

13. BGH 10 janvier 1996, DtZ 1996, 113.

14. BGH 26 janvier 1996, DtZ 1996, 138.

15. Voir O. Düsseldorf $1^{\text {er }}$ juin 1995, NJW 1996, 400, où fut jugé nul le contrat d'arbitrage, conclu par adhésion, et selon lequel le tribunal arbitral devait décider aussi de l'efficacité du contrat d'arbitrage quant à son dispositif (particulièrement concernant les clauses relatives au choix des arbitres ne permettent pas d'espérer un procès juste); voir BGH 9 novembre 1995, NJW 1996, 253, où on a refusé de reconnaître le caractère abusif d'un contrat d'assurance, étant donné sa clarté; voir BGH 6 décembre 1995, NJW 1996, 519, où on a refusé de reconnaître le caractère abusif d'un délai dans un contrat d'assurance; voir BGH 25 janvier 1996, NJW 1996, 1346, relatif à des clauses fiduciaires; voir BGH 7 mai 1996, 2032, sur des comptes postaux.

16. Voir BGH 29 mars 1996, NJW 1996, 1884, soulignant que, dans un contrat formel, la responsabilité précontractuelle ne pourrait surgir qu'en présence d'une grave violation des exigences de l'honnêteté.

17. Voir BGH 17 avril 1996, NJW 1996, 1967.

18. BGH 5 janvier 1995, BGHZ 128, 1996, 230 à la p. 236, où on a décidé que l'exigence d'un cautionnement à qui, n'ayant pas de moyens économiques suffisants, n'était pas en condition de bien comprendre les conséquences de son option; BGH 25 avril 1996, NJW 1996, 2088, où l'imprévision civile a été appliquée à un cautionnement entre conjoints, quand un divorce est survenu, et BGH 13 juin 1996, NJW 1996, 2369, avec un contrôle par l'article 242 BGB d'un cautionnement. La Cour Constitutionnelle elle-même s'est prononcée sur le contrôle judiciaire des cautionnements excessifs sans intervenir : BVerG 2 mai 1996, NJW 1996, 2021.

19. BGH 3 avril 1996, NJW 1996, 2100; après la fin du rapport contractuel, l'assureur n'est pas, ex bon fide, tenu de livrer des informations ultérieures et BGH 14 mai 1996, NJW 1996, 2092 : les garanties, devenues superflues, doivent être restituées.

20. Voir BGH 12 juin 1996, NJW 1996, 2367, dans le domaine de la dénonciation.

21. OLG Frankfurt 10 août 1995, NJW 1996, 1219. 
famille, ${ }^{22}$ sportifs $^{23}$ et de travail. ${ }^{24}$ La bonne foi est utilisée pour moraliser le fonctionnement des sociétés ${ }^{25}$ et pour délimiter les sphères de risque. ${ }^{26}$ Dans les nullités pour vice de forme, elle est appliquée de façon très restrictive. ${ }^{27}$

En France, l'article 1134 al. 3, du Code civil français jouissait, par tradition, d'une application limitée. Néanmoins, on observe une évocation plus fréquente de la bonne foi objective. ${ }^{28}$

On trouve des applications importantes de la bonne foi dans le contexte de la révocation unilatérale des contrats ${ }^{29}$ et dans un cas où on s'est livré à un

22. Voir BGH 12 avril 1995, BGHZ 129, 1996, 259 aux pp. 266-267 : l'imprévision peut être appliquée relativement à l'obligation alimentaire; voir également BGH 3 avril 1966, NJW 1996, 1894.

23. Voir BGH 28 novembre 1994, BGHZ 128, 1996, 93 à la p. 103 : les règles des associations sportives ne sont pas des conditions générales; toutefois, elles passent par le jugement de l'article 242 BGB.

24. Voir BAG 18 janvier 1995, NJW 1996, 805, sur l'extension du devoir de l'assureur de supporter des salaires, pendant une maladie.

25. Voir BGH 15 février 1996, NJW 1996, 1341 : le cautionnement, délivré par une société pour garantir un prêt du gérant lui-même à un débiteur dont il connaissait la piètre situation financière est contraire aux bonnes moeurs.

26. Voir OLG Nürnberg 27 juin 1995, NJW 1996, 1479, quant aux conséquences d'une invalidité survenue par une erreur mutuelle sur les aspects fiscaux d'un contrat.

27. BGH 20 mars 1996, DtZ 1996, 207 et BGH 2 mai 1996, NJW 1996, 1960.

28. A. Cimino, La clausula generale di buona fede nell' esperienza francese, (1995) XCIII RDComm, 785 à la p. 793 et G. Goubeaux, P. Bihr et X. Henry, Code Civil / Enrichi d'annotations tirées de bases de données juridiques (1995-96), art. 1134/C aux nos 112-121 (733), où sont citées plusieurs décisions de première instance. L'analyse des 31 numéros déjà parus du Recueil Dalloz Sirey en 1996 ne permet pas cependant de localiser des applications de l'article 1134 al. 3, du Code Napoléon ou de la bonne foi objective.

29. Cass. civ. $1^{\text {re }}, 31$ janvier 1995, Bull.civ.1995.I.41 au $\mathrm{n}^{\mathrm{o}} 57$ et commentaire J. Mestre, «Obligations en général» (1995) 94 Rev. trim. dr. civ. 618 aux pp. 623-624 : un couple achète une habitation, avec un prêt bancaire, de $200.000 \mathrm{~F}$., à payer en 15 ans. En octobre 1983, le mari, au chômage, a obtenu un moratoire jusqu'au $1^{\text {er }}$ février 1984, pour payer les prestations de novembre, décembre et janvier. Le 2 janvier 1984 la banque déclare au moyen d'une lettre qu'elle se trouve dans l'obligation d'exiger la restitution anticipée et intégrale du crédit; cependant, elle ne concrétise pas cette mesure; les emprunteurs payent l'essentiel de leur dette. Toutefois, le 2 juillet 1990, la banque interpelle le couple, pour le paiement de 91 $434 \mathrm{~F}$ correspondant à des intérêts et à des pénalités, étant donné la demeure. La Cour de Cassation a décidé que l'exercice dans ces conditions de la clause résolutoire était contraire aux exigences de la bonne foi. 
stratagème pour éviter le droit de préférence d'un éditeur. ${ }^{30}$ À l'exception de ces cas, la bonne foi n'est point utilisée dans les décisions des tribunaux.

En dépit du peu de place que le droit français réserve à l'application de la bonne foi, il faut dire que nous trouvons des décisions où l'on a recours à des concepts qui s'y apparentent. C'est ce qui se passe avec les clauses abusives, sanctionnées aux termes de la loi, ${ }^{31}$ quand elles donnent lieu à des déséquilibres graves, ${ }^{32}$ avec la responsabilité précontractuelle ${ }^{33}$ ou avec la responsabilité par l'apparence, ${ }^{34}$ dérivées de la faute.

\section{LES LECTURES CLASSIQUES}

Le rôle joué en pratique par la bonne foi permet de tirer quelques conclusions. Cependant, avant de passer à cette étape et de procéder à l'analyse des développements théoriques les plus récents, il paraît utile de rappeler l'état du droit lors des années quatre-vingts. ${ }^{35}$

30. Il s'agit d'une décision déjà ancienne (Paris 22 janvier 1992) mais publiée, seulement, en 1995 (J. Mestre, ibid. à la p. 624). Un contrat d'édition donnait à l'éditeur un droit de préférence dans l'édition de nouveaux ouvrages. Ce droit disparaissait s'il y avait deux refus de l'éditeur. L'auteur fit alors parvenir à l'éditeur deux manuscrits bizarres (un roman polyphonique et un roman dodécaphonique), objectivement impubliables et préparés manifestement en très peu de temps, pour neutraliser la préférence. La Cour a jugé ce comportement inefficace car contrevenant aux exigences de la bonne foi.

31. Il s'agit de l'article 131 al. 1 du Code de la Consommation, approuvé par la Loi no 93-949 du 26 juillet 1993, dans la rédaction donnée par la Loi n 95-96 du $1^{\text {er }}$ février 1995.

32. Cass. civ. $1^{\text {re }}, 31$ janvier 1995, Bull. civ. 1995.I.45 au n ${ }^{\circ} 64$, commentaire J. Mestre, supra note 29 à la p. 620 : une clause abusive ne cesse point de l'être, sous prétexte d'être fréquente et Cass. civ., 30 janvier 1996, D.1996. IR.59.

33. Versailles, 21 septembre 1995, Société Poleval c. Société Laboratoire Sandoz, $12^{\mathrm{e}} \mathrm{ch} .2^{\mathrm{e}}$ sect., commentaire J. Mestre, «Obligations en général» (1996) 95 Rev. trim. dr. civ. 143 à la p. 145, où on est arrivé à une indemnité de $4000000 \mathrm{~F}$, pour interruption injustifiée de négociations.

34. Cass. com., 28 juin 1994, Bull. civ. 1994.IV.190 $\mathrm{n}^{\circ}$ 243, commentaire J. Mestre, «Obligations en général» (1995) 94 Rev. trim. dr. civ. 93 à la p. 103 : une société avait comme objectif d'assurer la continuation d'une autre, en reprenant son activité et en achetant la totalité de ses actions à un prix symbolique; par des actions répétées, elle laissa penser à ceux qui avaient donné du crédit à l'entreprise en difficulté qu'elles seraient entièrement remboursée; il naît ainsi une confiance légitime de la part des créanciers qui, par le biais de la responsabilité civile, ont dès lors effectivement droit au remboursement.

35. Sur toute cette matière, voir le $1^{\text {er }}$ volume de notre ouvrage De la bonne foi en Droit civil (1985, en langue portugaise). 
La bonne foi, dans l'ancien droit romain, était déjà une création des juristes. Bien qu'étant à la base d'éléments religieux et sociaux antérieurs, la bonne foi a permis au préteur d'élargir la protection juridique à des situations tout à fait nouvelles, issues de l'expansion romaine dans la Méditerranée. Les jugements invoquant la bonne foi sont à l'origine, par exemple, des contrats d'achat, de louage, de mandat et de société, tels qu'on les connaît aujourd'hui.

Après avoir joué ce rôle créateur, la bonne foi, comme élément pacificateur et appréciatif, a été utilisée pour qualifier de nouvelles institutions et ce, par une diffusion horizontale. Telle est la situation de la personne qui, ignorant porter préjudice aux droits d'autrui, mérite une certaine protection de l'ordre juridique. Naquit ainsi la bonne foi subjective : le possesseur de bonne foi reçoit une certaine protection en fonction de son état d'esprit.

Dans la période de Justinien, la bonne foi a été encore une fois à l'origine de nouvelles créations juridiques. Elle s'est alors amalgamée avec des principes divers, comme le bonum et aequo et l'aequitas, constitués d'éléments empruntés à la rhétorique grecque. La notion se dilue, la bonne foi devient un principe général incapable, par son abstraction, de donner par lui-même des solutions concrètes. Parallèlement à ce principe survit un usage technique et précis de la bonne foi, dans les domaines possessoires et similaires : il s'agit de la bonne foi subjective - voir l'article 2919 du Code civil du Québec.

Le droit du Moyen Âge a apporté à la bonne foi deux importants renforts, l'un canonique, l'autre germanique. Le droit canonique donne alors à la bonne foi subjective un contenu éthique : est de bonne foi la personne qui a agi sans péché. Le droit germanique, quant à lui, réintroduit des éléments objectifs : la bonne foi est liée à la protection de l'apparence et au respect de la parole donnée.

Tous ces éléments ont pesé dans la pensée des jusnaturalistes, qui ont préparé les grands codes du Continent. Ils ont préservé une bonne foi subjective, à saveur éthique et psychologique, propre à la personne qui, sans faute, ignore porter préjudice à autrui : c'est la bonne foi subjective, typique de la possession et de la prescription et qui s'étend à d'autres concepts. Mais ils ont aussi donné une place à la bonne foi dans les contrats, visant ainsi à renforcer son pouvoir juridique. 
La tradition jusrationaliste a laissé des traces claires dans le Code civil français. À côté de la bonne foi subjective, confinée à certains domaines, nous trouvons une évocation très brève de la bonne foi objective à l'article $1134 \mathrm{al}$. 3 , en renfort du rapport contractuel. Toutefois au $\mathrm{XIX}^{\mathrm{e}}$ siècle, l'idée du respect des contrats était tout à fait assurée; il n'était pas nécessaire de la doubler, par la bonne foi. Le notion est appliquée modérément, on en oublie même l'origine.

En Allemagne, l'évolution fut différente. Son unification tardive retarde la préparation du Code civil, qui ne voit la lumière qu'à la toute fin du XIX ${ }^{\mathrm{e}}$ siècle. Pendant tout le $\mathrm{XIX}^{\mathrm{e}}$ siècle, les tribunaux et les juristes allemands doivent vivre sur la base du Corpus Iuris Civilis. En face de problèmes tout à fait nouveaux, surtout du domaine commercial, les tribunaux développent une technique pour résoudre les problèmes, raisonnablement, faisant appel à la vieille bona fides. Ainsi apparaît une tradition particulière, qui se loge à l'article 242 du BGB naturellement.

Dès le début, l'article 242 BGB facilite le succès de quatre notions importantes : la culpa in contrahendo, les devoirs accessoires, l'abus de droit et l'imprévision.

La culpa in contrahendo postule qu'avant la conclusion d'un contrat et durant les négociations préliminaires, les parties doivent observer des devoirs de sûreté, d'information et de loyauté. Au début, il s'agissait d'éviter que des gens soient lésés. Plus tard, la notion évolue dans le sens de la protection de la partie défavorisée : la plus forte doit, par la loyauté et l'information, éviter que la faiblesse de son partenaire puisse se traduire par des déséquilibres contractuels.

Les devoirs accessoires nous rappellent que, dans les contrats, il s'agit effectivement de réaliser les avantages reconnus au créancier. Ainsi, le débiteur, au nom de la bonne foi, est obligé aux comportements instrumentaux nécessaires à l'intégralité de la prestation principale. Ici aussi, il est possible de déterminer des devoirs de protection, d'information et de loyauté.

L'abus de droit - ou, plus largement, l'exercice inadmissible d'avantages juridiques - celui qui occupe la positon la plus favorable à s'en prévaloir dans le cadre du système. Ainsi, il ne peut pas agir contre les expectatives qu'il a contribué à créer - venire contra factum proprium; il ne pourrait pas non plus 
se prévaloir des violations que, lui-même, aurait perpétrées - tu quoque - ou agir, sans avantages propres, rien que pour porter préjudice aux tiers - exercice en déséquilibre.

Finalement, l'imprévision établit que quoique les contrats librement conclus doivent être exécutés jusqu'à la limite de l'impossibilité, leur exécution ne peut être exigée quand les circonstances qui ont accompagné leur conclusion se sont profondément modifiées. La base juridique - tout comme à propos des trois autres notions - en est la bonne foi et l'article $242 \mathrm{du}$ BGB.

La législation actuelle de protection du consommateur, particulièrement dans le domaine des conditions générales des contrats, a recours à la bonne foi comme critère ultime pour la détermination des clauses abusives.

L'évolution française a été, dans ces domaines, assez différente. Quelques unes des notions fondées en Allemagne sur la bonne foi n'ont jamais été admises en France. C'est le cas de l'imprévision civile. D'autres concepts ont été élaborés, mais par le biais de la responsabilité civile. C'est le cas de la culpa

in contrahendo, dérivée de la faute précontractuelle et de l'abus de droit, développée - du reste, de façon limitée - aussi sur la base de la faute.

Ce n'est que récemment et - probablement - grâce à une certaine influence doctrinale externe, que nous observons des applications de la bonne foi prévues à l'article 1134 al. 3 du Code civil français. Ainsi apparaissent les devoirs de loyauté ou les interdictions de détourner le sens véritable et ultime des contrats. bonne foi.

La protection des consommateurs n'a pas, pour sa part fait appel à la

\section{L'IMPÉRATIF DE LA CONFIANCE ET LA MATÉRIALITÉ SOUS-JACENTE; LES SYSTÈMES DE LA BONNE FOI ET DE LA FAUTE}

On peut trouver, sous-jacents aux concepts qui se réclament de la bonne foi, deux grands principes : l'impératif de la confiance et la primauté de la matérialité sous-jacente. 
L'impératif de la confiance accorde une certaine protection à la personne qui, en vertu du comportement d'une autre, est induite à croire à la conservation d'un certain état des choses. En principe, on exige que la croyance soit légitime, qu'en celle-ci le confiant ait dépensé des énergies matérielles ou personnelles et que le tout soit imputable à celui qui, après, va être obligé de respecter la situation créée.

La primauté de la matérialité sous-jacente rappelle que le droit ne se limite point à des actions rituelles : il tend, en vérité, à la poursuite de certaines valeurs, qui sont sous-jacentes aux normes juridiques. Sont donc contraires à la bonne foi les comportements qui ne font que respecter l'extériorité formelle du droit, en méprisant ses valeurs les plus profondes.

Au fond, nous pouvons étendre l'impératif de la confiance à l'exigence de l'égalité : on ne peut traiter celui qui s'est retrouvé dans une situation de confiance et qui, sur elle, a édifié toute une partie de sa vie juridique et personnelle, comme si de rien n'était. L'égal doit être traité d'une façon égale et le différent de façon différente, selon la mesure de la différence.

La primauté de la matérialité fait appel à l'idée de droit, en tant qu'ordre humain effectif.

Force nous est donc de conclure que, par la bonne foi, on peut introduire en chaque décision juridique la possibilité et la nécessité de donner corps aux données profondes du système.

Cependant, il faut remarquer que dans les ordres juridiques du Continent il y a deux systèmes différents : ceux qui font appel à la bonne foi de façon explicite et ceux qui atteignent des résultats semblables grâce à un type particulier de responsabilité, tiré de la notion de «faute».

Le système de bonne foi par excellence est celui de l'Allemagne. Des raisons historiques et culturelles déjà remarquées démontrent que, dès le début, les tribunaux et la doctrine avaient procédé à la concrétisation de la notion. 
Le système français a trouvé des solutions semblables grâce à une conception particulièrement flexible de la responsabilité civile. ${ }^{36}$ Celle-ci tient à un pré-requis primordial : l'existence d'une faute. La référence à la faute devient intuitive, de telle façon qu'elle dispense (de) l'indication concrète des normes juridiques violées. Devant le juge français, il est possible de se pourvoir à l'encontre d'une situation qui n'est point prévue par le droit strict, en invoquant la faute de quelqu'un.

Les divers droits de l'Europe ont évolué à partir de leurs propres traditions et, encore, sous des influences culturelles et juridiques. Au XIX siècle, l'influence du Code civil français fut marquante. Plus tard le système allemand gagna du terrain. Nous en avons des exemples dans les codes suisse, italien et portugais.

\section{TENDANCES RÉCENTES : LA RÉDUCTION DU RÔLE DE LA BONNE FOI}

Les tendances doctrinales plus récentes, selon notre opinion, conduisent à la réduction de la place qui était reconnue à la bonne foi. ${ }^{37}$ Elle ne fonctionne plus comme source de notions tout à fait nouvelles : on lui attribue plutôt des fonctions de concrétisation, de complémentarité, de limitation et de correction. ${ }^{38}$

Cette évolution existe tout simplement parce que les concepts dérivés de la bonne foi ont gagné leur propre autonomie. Ils ont des règles spécifiques stables et n'ont point besoin d'en appeler à la bonne foi d'origine, sauf quant à leur histoire.

Ainsi en est-il de la culpa in contrahendo (ou responsabilité précontractuelle). Concept juridique achevé, attestant du succès de la bonne foi, la culpa in contrahendo se concrétise aujourd'hui en des devoirs clairs de

36. Quant à la genèse du paradigme français de la responsabilité civile, fondé sur la faute par opposition au droit allemand, issu d'une dichotomie entre culpabilité et d'illécéité, voir notre ouvrage De la responsabilité civile des administrateurs des sociétés commerciales (1996, en langue portugaise), $\S 18^{\circ}$.

37. Un aperçu des interprétations existantes, sur l'article 242 du BGB : J. Schmidt, dans le Staudingers Kommentar zum BGB, 13e éd., 1995, Nr. 113 aux Nr. 268 et s.

38. Ainsi l'évolution de Palandt/Henrichs, $B G B, 39^{\mathrm{e}}$ éd., 1980 à l'article 242 et $B G B, 55^{\mathrm{e}}$ éd., 1996 à l'article 242. 
protection, d'information et de loyauté. Ces derniers se fondent sur des décisions judiciaires stables et mûries. Il n'est plus nécessaire de retourner aux données fondamentales du système et d'évoquer la bonne foi. Les devoirs précontractuels sont fixés : leur violation entraîne une responsabilité de type contractuel et, en certains cas, une responsabilité délictuelle. ${ }^{39}$

Les divers devoirs de comportement, dérivés, jadis, dans des situations de proximité de la bonne foi, vont désormais s'appuyer sur des pondérations d'intérêts face aux valeurs typiques en présence. ${ }^{40}$

L'impératif de la confiance a d'ailleurs généré l'établissement d'une technique propre dès les années soixante-dix et ce, grâce aux travaux de Claus-Wilhelm Canaris. ${ }^{41}$

L'imprévision est aujourd'hui fortement dépurée par l'intervention de plusieurs concepts. ${ }^{42}$ Malgré la reconnaissance de son rôle dans l'encadrement des questions nouvelles posées par la réunification allemande, ${ }^{43}$ l'imprévision, comme notion, est cantonnée dans des secteurs spéciaux. ${ }^{44}$ Des problèmes qu'elle contribuait jadis à résoudre sont aujourd'hui résolus par le recours au régime de l'erreur, aux règles générales ou spécifiques du risque ou aux principes de l'interprétation des contrats.

39. N. Horn, Culpa in contrahendo, JuS 1995, 377.

40. J. Hennrichs, Treupflichten im Aktienrecht/zugleich Überlegungen zur Konkretisierung der Generalklausel des $\$ 242$ BGB sowie zur Eigenhaftung des Stimmrechtsvertreters, AcP 195, 1995, 221 aux pp. 271 et 272. La doctrine souligne de toute façon la nécessité de ne pas survaloriser les devoirs de loyauté sociétaires : L. Häsemeyer, Obstruktion gegen Sanierung und gesellschaftsrechtliche Treupflichten, ZHR 160, 1996, 109 à la p. 132.

41. En particulier : C.-W. Canaris, Die Vertrauenshaftung im deutschen Privatrecht (1971).

42. À propos de la clausula, Abas préconise la limitation de la bonne foi : P. Abas, Rebus sic stantibus (1993), XIII. Quant à la dépuration de l'imprévision, voir M. Cordeiro, Conventions de travail et imprévision (1995, en langue portugaise), aux pp. 80 et s., avec des indications.

43. S. Görk, Deutsche Einheit und Wegfall der Geschäftsgrundlage (1995).

44. I. Koller, Dei Bedeutung des Haftungsmassstabs für die Zurechnung des Leistungserschwerungsrisikos, NJW 1996, 300 à la p. 301. 
Finalement, le domaine de la protection des consommateurs dispose d'ores et déjà d'une dogmatique propre, ${ }^{45}$ du reste accompagnée d'une interférence communautaire européenne croissante; ${ }^{46}$ la bonne foi ne subsiste que comme porte de secours pour des questions limitées et inattendues.

Dans l'exposé fondamental de Jürgen Schmidt, les normes nouvelles, d'abord légitimées par la bonne foi, sont étudiées selon leur position dans le système général du BGB. ${ }^{47}$

Nous pouvons ainsi avancer une première conclusion. La bonne foi a engendré plusieurs notions et concepts juridiques nouveaux : probablement les créations juridiques les plus stimulantes et avancées des deux derniers siècles. Ce rôle créateur terminé, ces notions et concepts nouveaux se regroupent dans le système selon les réalités auxquelles ils ont trait. Ils reçoivent un traitement de plus en plus proche du droit strict. La bonne foi se confine dès lors tout juste dans un espace restreint, où l'intervention normalisatrice de la science du droit n'a pas été encore possible.

Il s'agit d'une conclusion qui, probablement et selon nous, ira mettre un terme à la fin du siècle, à l'empire du «paragraphe royal»du BGB allemand. Cette échéance serait d'autant plus surprenante qu'elle coïnciderait avec l'ouverture du droit français à la bonne foi. En sera-t-il ainsi?

\section{LA MÉTHODE : UNE AIDE PRATIQUE?}

Le rapprochement de plusieurs notions - quoique dans une dimension de plus en plus historique -et de la bonne foi pose des problèmes de méthode. $\mathrm{Ce}$ n'est qu'en considérant un problème spécifique que nous pouvons le préciser en

45. M. Wolf et C. Ungeheuer, Zum Recht der allgemeinen Geschäftsbedingungen, JZ 1995, 77 et 176, J. Schmidt--Salzer, Recht der AGB und der missbräuchlicher Klauseln : Grundfragen, JZ 1995, 223 et D. Medicus, Schutzbedürfnisse (insbesondere der Verbraucherschutz) und das Privatrecht, JuS 1996, 761.

46. W. Nassal, Die Anwendung der EU-Richtlinie über missbraüchliche Klauseln in Verbraucherverträgen / Zugleich ein Beitrag zur Auslegung gemeinschaftsrechtlicher Generalklauseln, JZ 1995, 689, H. Hennrichs, Das Gesetz zur Änderung der AGB-Gesetzes, NJW 1996, 2190 et K. Tonner, Die Rolle des Verbraucherrechts bei der Entwicklung eines europäischen Zivilrecht, JZ 1996, 533 à la p. 533 et passim.

47. J. Schmidt, supra note 37 au Nr. 322-323 (335) et 324 et s. (335 et s.) 
recourant à des valeurs et des arguments destinés à l'élaboration des modèles de décisions nécessaires. Les divers concepts qui trouvent leur origine récente dans la bonne foi présentent, aujourd'hui encore, une indétermination d'origine. En face de propositions indéterminées, on demande à la science du droit un effort redoublé de précision et de clarté.

Qu'est ce que la méthodologie juridique nous donne à ce niveau? Pourrait-on redonner un rôle de dirigeant unitaire à la vieille bona fides?

La pondération des courants méthodologiques les plus récents, avec un relief particulier pour la jurisprudence analytique, la jurisprudence problématique et les synthèses herméneutiques, ne permet point de découvrir des influences pratiques. On en arrive, ainsi à un irréalisme méthodologique facile à décrire : après la vieille jurisprudence des intérêts, la pensée méthodologique n'a pu déterminer d'autres modèles capables d'intervenir dans le cas concret. ${ }^{48}$

Au cours des toutes dernières années, nous croyons découvrir quelques signes qui permettraient de pallier à l'irréalisme méthodologique et ceci, sans tomber dans un positivisme de recours. Ces signes pointent sur une pensée systématique ouverte, mouvante, hétérogène et cybernétique. Avant de vérifier ces possibilités, nous allons étudier deux propositions qui, récemment, sont apparues dans le domaine de la concrétisation de la bonne foi : le contenu éthique de la bonne foi et le rôle de l'économique dans la concrétisation juridique.

La référence à un contenu éthique de la bonne foi émerge surtout dans la littérature juridique française renouvelée, de la bona fides. ${ }^{49}$ La distinction entre morale et droit est classique : nous n'allons pas la reprendre. Où que l'on

48. Vide notre ouvrage Science du Droit et Méthodologie Juridique à la Fin du XX Siècle (1989, en langue portugaise), 15 et s. L'irréalisme est confirmé par l'observation. Ainsi H. Rüssmann, en recension de Larenz et Canaris, Methodenlehre der Rechtswissenschaft, $3^{\mathrm{e}}$ éd., 1995, NJW 1996, 1264, révèle que dans une recherche par logiciel sur 90000 décisions de tribunaux allemands en texte intégral seulement 285 mentionnaient le terme Methodenlehre (méthodologie), dont 265 à propos du livre Methodenlehre, de Larenz. Il nous semble évident que tout ouvrage méthodologique sera irréel - dans le domaine du droit, comme science du cas - s'il n'est pas lu et utilisé par les tribunaux dans le domaine des décisions. 
soit, quant à la discussion philosophique, nous savons aujourd'hui que la morale et le droit sont transmis aux personnes de l'extérieur, tout au long de leur développement : c'est la socialisation, au sens sociologique. Cet apprentissage se fait de façon indifférencié. Si les données diverses sont prises isolément, rien ne sépare les règles juridiques des règles morales. Nous ne pouvons pas faire appel aux sanctions -et, en particulier, à la coercition - pour isoler le droit : les normes imparfaites prolifèrent, tandis que se multiplient les normes dont la sanction est douteuse ou d'application difficile. Justement, dans ces situations de défaut de sanction, il faut très clairement affirmer la nature juridique des commandements.

Le droit se sépare de la morale par son positivisme. Il s'organise comme un corps de règles posées - posita -de façon organisée et assorties d'instances d'apprentissage et d'application. Nous pouvons en déduire deux conclusions de la plus grande importance :

- le droit est toujours beaucoup plus développé que la morale; celle-ci s'épuise dans de grands principes ou règles immédiates, inapplicables dans des situations plus complexes; ainsi, quand il n'y a pas de solution juridique, il est inutile de s'adresser à la morale, plus pauvre - en ce sens - que le droit;

- le droit - c'est à dire le droit véritable -coïncide avec la morale dans ses jugements : la solution immorale est, à coup sûr, injuste et probablement illicite.

La faiblesse de la morale est sa plus grande force : elle ne dépend pas d'instances d'application, mais de la conscience de la personne (morale individuelle) ou du sentiment social (morale sociale). On peut tromper l'instance d'application juridique; mais pas l'«instance» morale.

Retournons, avec ces éléments, à la bonne foi. Il s'agit d'une notion juridique : elle surgit pour se concrétiser selon des lignes juridiques et, tout particulièrement, par décision des tribunaux. En l'absence de vecteurs juridiques, pour la concrétiser, il ne paraît guère réaliste de se tourner vers la morale. Et si, par hasard, nous trouvons des arguments éthiques, il n'est point pensable que l'on ne puisse pas procéder à leur «positivisation», en les élevant 
à des postulats de droit. Il pourra donc y avoir une «bonne foi éthique»; mais la morale ne permet pas la concrétisation de la bonne foi juridique.

Le rôle de l'économie dans le droit est souligné dans divers domaines. La responsabilité civile est particulièrement en cause, surtout dans la mesure où l'on vérifie son «instrumentalisation» au service des valeurs extra civiles ${ }^{50}$ : l'économie, la défense de l'environnement, la sécurité ou la protection des personnes. La bonne foi elle-même pourrait être facilement visée : dans un droit au service d'objectifs concrets -nommément économiques dans un monde comme le nôtre - l'économique pourrait dicter le sens des règles à observer.

Cette piste -désolante d'ailleurs pour le droit - n'est guère opérationnelle. L'économique est riche en abstractions, travaillant sur des hypothèses. Il nous donne de grands topiques. Mais il aide très peu dans les cas concrets. ${ }^{51}$ Nous restons seuls encore une fois.

Dans le droit comme dans d'autres domaines, il faut être prudent. Tout indique cependant l'insuffisance des méthodologies, incapables de résoudre les problèmes ou de les prendre comme base de réflexion. À la question : «qu'est-ce que la méthodologie peut faire pour le droit pratique et, tout particulièrement, pour la bonne foi?», on pourra répondre : que peut faire le droit pratique - et donc la bonne foi - pour la méthodologie?

\section{BILAN : LE RÔLE ACTUEL DE LA BONNE FOI; PROBLÈMES ET PENSÉE SYSTÉMATIQUE}

Le rôle de la bonne foi s'analyse en de multiples propositions; il peut subir des traitements par des sensibilités diverses. ${ }^{52}$ Pour les fins de cet étude, nous nous limiterons aux considérations qui suivent.

La bonne foi a légitimé l'interprétation créative du droit, qui a soutenu des notions telles que la culpa in contrahendo, les devoirs accessoires, l'abus

50. C. Engel, Zivilrechte als Fortsetzung des Wirtschaftsrechts mit anderen Mitteln, JZ 1995, 213 à la p. 215.

51. J. Taupitz, Ökonomische Analyse und Haftungsrecht - Eine Zwischenbilanz, AcP 196, 1996, 114 aux pp. 165 et 166.

52. Il s'agit d'une matière très bien traitée en dernier lieu par J. Schmidt, supra note 37 aux pp. 306 et s. et passim. 
de droit ou l'imprévision. La bonne foi a donc permis la concrétisation de ces notions, nécessairement créées au terme d'une grande indétermination. La bonne foi a facilité des corrections ponctuelles à des concepts de droit strict. Dans les pays où le législateur lui-même a introduit de façon directe les grandes créations de la bonne foi - l'Italie, le Portugal, le Québec -celle-ci se limiterait, naturellement, aux rôles de concrétisation et de connexion.

La pondération de la casuistique la plus récente sur la bonne foi nous montre qu'elle maintient intacte une capacité d'intervention en face de problèmes nouveaux, privés en un premier temps, de solutions strictes ${ }^{53}$ : En effet, le cycle relatif à la réunification allemande nous montre comment, sans sursauts, elle a su légitimer des solutions équilibrées, et reconnues par le système, comme étant les siennes.

La bonne foi s'affirme aussi par un élargissement de solutions préexistantes : pensons, par exemple, aux rapports de famille ou aux règles des associations sportives, syndiquées ou corrigées par le système par le biais de la bonne foi.

Finalement, la bonne foi prête son langage et son pouvoir de consensus aux équilibres, encore instables, sollicités au nom de la défense des consommateurs.

C'est indubitable, le noyau laissé à la bonne foi pure est aujourd'hui considérablement réduit. Cependant, aucun système n'osera se proclamer tellement autosuffisant qu'il puisse immédiatement se priver de la possibilité de réduire des questions nouvelles sans intervention du législateur.

La bonne foi est à l'origine de notions et de concepts qui sont aujourd'hui autonomes. L'origine de ces derniers leur donne toutefois un sens particulier. Dans le système, ils vont aussi être placés en fonction de ce qu'ils ont représenté et représentent : une concrétisation particulière, en face de questions pré-systématiques, de données basiques du système lui-même. Par exemple : les devoirs précontractuels perdurent pendant les contrats, comme devoirs

53. Àce propos, voir U. Burgard, Das Wirksamwerden empfangsbedürftiger Willenserklärungen im Zeitalter moderner Telekommunikation, AcP 195, 1995, 74 à la p. 134 : l'appel à la bonne foi pour fixer l'efficacité des communications transmises par les moyens actuels. 
accessoires et peut-être même après leur extinction, dans des phénomènes d'efficacité post-contractuelle. Il peut y avoir un traitement commun, sur la base des références à la bonne foi. Un autre exemple : l'imprévision est limitée par l'interprétation élargie des contrats qui implique donc une interprétation «selon la bonne foi», par l'impératif de la confiance et par l'exercice inadmissible de certaines positions juridiques; là aussi s'impose une gestion globale de tous ces aspects.

En somme, les conceptions juridiques dérivées de la bonne foi retournent à leur racine - i.e. la bonne foi - pour se perfectionner dans leur fonctionnement pratique. La science du droit, sur la base d'un traitement systématique, corrige des asymétries, recherche des solutions, complète des encadrements et présente des décisions plus différenciées et mieux justifiées. La bonne foi conserve donc un excellent pouvoir juridique, même en face de questions déjà connues.

Le droit est fortement conditionné par le langage. Or, dans les contacts entre les peuples et surtout dans les mouvements d'intégration, le problème des références communes se pose : il faut trouver des lieux communs, où tous peuvent se reconnaître. La bonne foi est utile dans ce domaine : son rôle dans les instances communautaires européennes est reconnu. ${ }^{54}$

Dans toutes ses applications, la bonne foi - tout comme la généralité de ses concrétisations - n'énonce pas de propositions abstraites : elle résout des problèmes, avec ses solutions. Quelques considérations supplémentaires s'imposent.

L'idée de problème - ou cas d'application, cas exemplaire, cas typique, problème exemplaire typique ${ }^{55}$ - suscite une pré-compréhension très importante du sujet qui nous intéresse. Il faut toutefois aller plus loin en isolant autant que possible ses contours, son contenu et sa fonction.

54. Zimmermann, supra note 3 à la p. 490 et R. Knütel, Ius commune und römisches Recht vor Gerichten der Europäischen Union, JuS 1996, 768 aux pp. 773 et 774.

55. C.-W. Canaris, Théorie et cas d'application dans la Science du Droit, version française de la leçon donnée à l'Université de Lisbonne, à propos de son doctorat honoris causa (1990, inédit), en partie repris dans l'article Funktion, Struktur und Falsifikation juristischer Theorien, JZ 1993, 377 aux pp. 384 et s. 
Le problème ou cas typique n'est pas un cas concret. Souvent, il peut tenir compte, dans sa formulation, de circonstances historiquement vérifiées. Cependant, alors que celles-ci sont illimitées dans la complexité de leurs prémisses - prémisses sociales, personnelles, historiques, géographiques, climatiques, économiques, politiques et juridiques par exemple -, le problème ou cas typique repose sur des propositions limitées et maniables. On supprime du cas concret tout ce qui dans la conjoncture en cause se présente comme non nécessaire, pour ne conserver que l'essentiel. Ou, dans l'hypothèse d'un problème artificiel (i.e. académiquement conçu), on opère dès le début avec un nombre limité et pré-sélectionné de prémisses problématiques.

Le problème ou cas typique n'est point une hypothèse abstraite. En le confrontant au cas concret, le problème est sans doute capable d'englober plusieurs cas concrets grâce à la simplification qu'il opère. Dans ce sens, ce serait une abstraction. Mais les prémisses dans lesquelles le problème repose ne sont point des formulations abstraites : elles correspondent à des données effectivement descriptives de la réalité, délivrées de compléments inutiles, et qui ne sont pas indispensables à la solution recherchée.

Le problème ou cas typique ne se confond pas avec le modèle de décision : contrairement à celui-ci, le problème contient sa propre solution. Tous les deux indissociables, ils communiquent un certain sens de réalisation du droit.

Il n'y a pas de règles quant au nombre minimal de propositions pour faire un problème. L'interprète-applicateur a ainsi les mains libres pour composer des problèmes plus ou moins denses, selon les objectifs qu'il se propose. Les limites minimales seront posées, dans la pratique, par les nécessités de cohérence et de viabilité, relatives aux solutions à trouver. La critique des solutions et leur justification assureront la solidité du tout.

Utilisé dès les débuts pour comprendre, expliquer et justifier le droit, le problème typique gagne du terrain. Il suffit de voir qu'il a une résolution plus étroite avec un maximum d'informations : celles-ci pourraient être transmises seulement avec des propositions abstraites.

Le problème est encore une fois très utile dans certains domaines interdisciplinaires et face aux questions nouvelles. On devine, sous ces considérations, l'importance de la discussion des cas. 
La bonne foi gagne son ampleur dans une pensée systématique renouvelée. Le système doit être ouvert : à l'extérieur, parce qu'il admet des questions qui lui étaient étrangères et que la bonne foi a d'ailleurs permis de découvrir, solutionner et ensuite intégrer dans le système; à l'intérieur parce qu'il accueille des éléments étrangers, expliqués aussi par la bonne foi : pensons à l'abus de droit. Le système est malléable : les éléments qui le composent ne se trouvent pas dans des hiérarchies rigides, tout en étant compensables. Le système est hétérogène : il est évident - la bonne foi le démontre - qu'il y a des zones de densité variable, y compris des failles dans le système et des lacunes rebelles à l'analogie. Le système, enfin, est cybernétique : il est attentif aux conséquences de ces solutions et il les modifie en fonction des impressions périphériques qu'il reçoit.

La pensée juridique doit donc reposer sur l'étude et la méditation des cas, des règles et des solutions - ou elle ne sera plus juridique du tout.

\section{LE FUTUR}

Aucun juriste n'irait prendre le risque de prophétiser par écrit le futur de sa propre science. ${ }^{56}$ Toutefois, en considérant le présent immédiat on pourrait peut-être avancer quelque chose : l'expérience nous enseigne que malgré toutes les nouveautés le droit civil présente une stabilité très marquée, qui n'est comparable qu'au langage et ce, même dans les domaines sensibles comme celui de la bonne foi.

En ce moment, le BGB allemand célèbre sont premier centenaire ${ }^{57}$ alors que le Code civil français s'approche rapidement du second. L'éventualité d' une réforme, voire d'une substitution intégrale, semble révolue. Les tentatives de réforme, particulièrement du BGB, qui se sont prolongées dans toute la décade de quatre-vingts ont eu le mérite de démontrer que, tout compte fait, la science du droit fonctionne à tout moment sans devoir modifier ses sources. ${ }^{58}$

56. D. Joost, Eine vergangene Zukunft der Rechtswissenschaft, JZ 1995, 11 à la p. 11.

57. M. Schmoeckel, 100 Jahre BGB : Erbe und Aufgabe, NJW 1996, 1697 et R. Stürner, Der hundertste Geburtstag des BGB - nationale Kodifikation im Greisenhalter?, JZ 1996, 741. Quant aux origines : H. Schulte-Nölke, Die schwere Geburt des Bürgerlichen Gesetzbuchs, NJW 1996, 1705.

58. B.-R. Kern, Neuere Entwicklungen im Bürgerlichen Recht, JuS 1996, 1 à la p. 2. 
Nous pouvons aussi conclure que le droit civil a tenu ferme face à l'impact de la seconde moitié du $\mathrm{XX}^{\mathrm{e}}$ siècle : celui du commerce de masse. ${ }^{59}$ Des mesures efficaces pour la défense du consommateur et du contractant en position de faiblesse - toujours la bonne foi! - ont été prises; il est certain que, des divers modèles développés dans ce domaine, les solutions «privées» se sont révélées les plus efficaces. Il ne paraît pas trop risqué de prévoir le maintien et le renfort de cette tendance.

Il y a clairement en ce moment une lacune en ce qui touche la défense des personnes. Il faudrait, dans ce domaine, un équivalent moral - c'est-à-dire non patrimonial - de ce que la bonne foi représente dans le champ patrimonial. C'est un rôle qui pourrait être joué par les droits fondamentaux dès qu'ils seront «civilisés» et donc définitivement soulagés de la turbulence qu'aujourd'hui encore représentent les affirmations politiques.

L'intégration européenne se prête à des équivoques. Nous ne voyons aucune utilité ni aucun mérite dans l'établissement de codes privés européens : il n'y a pas de nécessité économique qui puisse l'exiger; il n'y a pas de critère de choix des solutions; il n'y a pas non plus de science unitaire qui tienne ce pari et nous aurions une inconcevable perte culturelle. Tout au plus, nous pourrions mettre à l'oeuvre une lex mercatoria ${ }^{60}$ en des points limités. L'unité européenne devra fonctionner avant tout comme une garantie supplémentaire de liberté et ce, même - et surtout - dans le droit de chaque État. ${ }^{61}$ Tout le Monde est d'accord quant au sens basique de la bonne foi. Cependant, la comparaison des expériences allemande et française nous montre bien le désastre que serait l'oubli de l'une d'elles en faveur de l'autre. L'Histoire moderne nous montre comment des peuples minoritaires, parfois dans des situations difficiles, intégrés dans des communautés riches, puissantes et de haut niveau culturel, ont su, malgré l'intégration politique, sociale et économique pleine, préserver et renforcer leur droit : la Louisiane et le Québec. Le premier de ces deux cas nous montre même que, soutenu par la culture de ses juristes, le droit est plus résistant que la langue elle-même.

59. O. Sandrock, Das Privatrecht am Ausgang des 20. Jahrhunderts : Deutschland - Europaund die Welt, JZ 1996, 1 à la p. 1

60. Ibid. aux pp. 7 et 8 .

61. P. Von Wilmowsky, EG - Freiheiten und Vertragsrecht, JZ 1996, 590. 
Les droits nationaux vont subsister. Sur eux surgit toutefois le spectre de l'abâtardissement. On ne pourra lutter contre lui qu'en lui opposant la qualité des juristes.

Dans les sociétés, on en veut aux juristes. ${ }^{62}$ Ils représentent un obstacle à la concrétisation immédiate des volontés individuelles et collectives, tout en passant inaperçus quand ce n'est pas le cas. Toutefois, à mesure que les sociétés deviennent plus interdépendantes et que l'ambition du présent cache les risques de l'avenir, les juristes doivent leur rôle accru, dans la préservation de la culture et des biens les plus personnels. Il faut le dire. ${ }^{63}$ Une activité plus visible et plus efficace, avec du protagonisme s'impose. L'interdisciplinarité et l'internationalisation agissent, paradoxalement, comme des conditions de sauvegarde des particularismes.

La bonne foi surgit encore une fois dans d'excellentes conditions, pour reprendre son infatigable - et très efficace - rôle de perpétuation du système : elle peut le renouveler dès que nécessaire. Son futur est assuré. D'ailleurs il est déjà là.

62. J. Brau, Über die Unbeliebtheit der Juristen, JuS 1996, 287.

63. E. Schmidt-Assmann, Zur Situation der rechtswissenschaftlichen Forschung, JZ 1995, 2. 